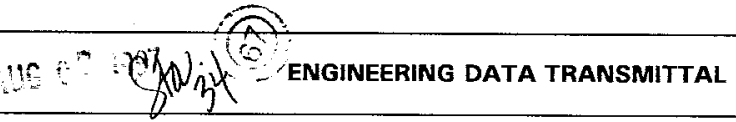

1. EDT 618806

\begin{tabular}{|l|}
\hline 2. To: (Receiving organization) \\
Structural Engineering \\
\hline 5. Proj./Prog./Dept./Div.: \\
L-274 - HSEAS
\end{tabular}

8. Originator Remarks:

This transmittal transmits for approval the calculations and drawing for a monopole for the siren system. The calculations and drawing are vendor supplied and need reviewing and acceptance by structural engineering.

11. Receiver Remlarks: 11A. Design Basel ine Document? [] Yes

\author{
3. From: (originating Organization) \\ Electrical Engineering \\ 6. Design Authority/ Design Agent/Cog. \\ Engr.: \\ Jeff What tam
}

\section{Related EDT No.: $N / A$ \\ 7. Purchase Order No.: \\ MV5-SPD-83267}

9. Equip./Component No.: $\mathrm{N} / \mathrm{A}$

10. System/Bldg./Facility: Sitewide system

12. Major Assm. Dwg. No.: $N / A$

13. Permit/Permit Application No.: N/A

14. Required Response Date: Nov 1, 1996

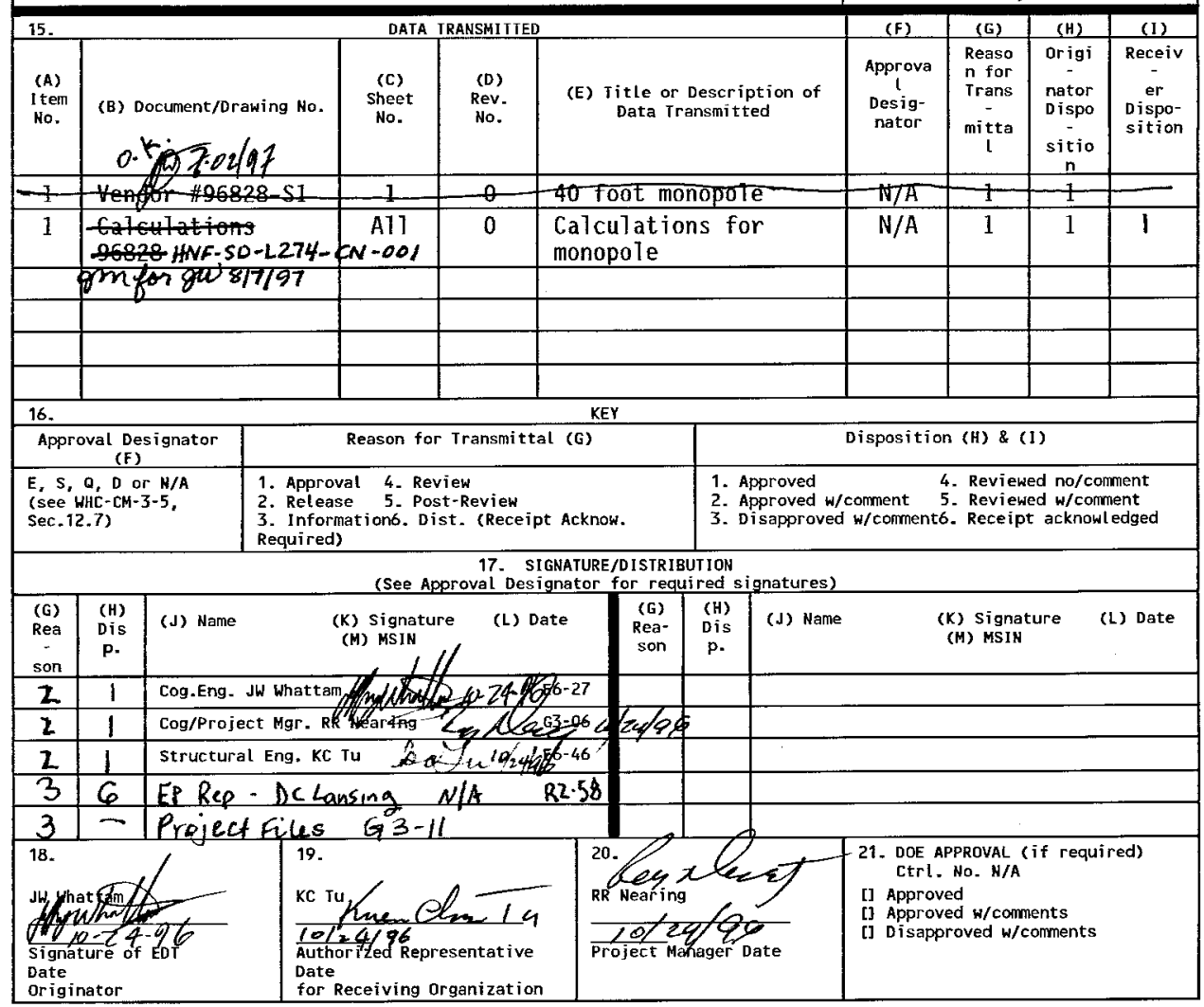




\title{
Calculations for the 40' Monopole for the Hanford Site Emergency Alerting System
}

JW Whattam

FDNW, Richland, WA 99352

U.S. Department of Energy Contract DE-AC06-96RL13200

\author{
EDT/ECN: $618806 \quad$ UC: 600 \\ Org Code: 57300 Charge Code: FE274 \\ B\&R Code: EW7070201 Total Pages: 15

Key Words: Calculations, monopole, HSEAS, Hanford site emergency a)erting system

Abstract: This document provides calculations for the $40^{\prime}$ monopole used for the Hanford site emergency alerting system.

TRADEMARK DISCLAIMER. Reference herein to any specific comercial product, process, or service by trade name, trademark, manufacturer, or otherwise, does not necessarily constitute or imply its endorsement, recormendation, or favoring by the United states Government or any agency thereof or its contractors or subcontractors.

Printed in the United States of America. To obtain copies of this document, contact: Document Control Services, P.O. Box 950, Mailstop H6-08, Richland HA 99352, Phone (509) 372-2420; Fax (509) $376-4989$.
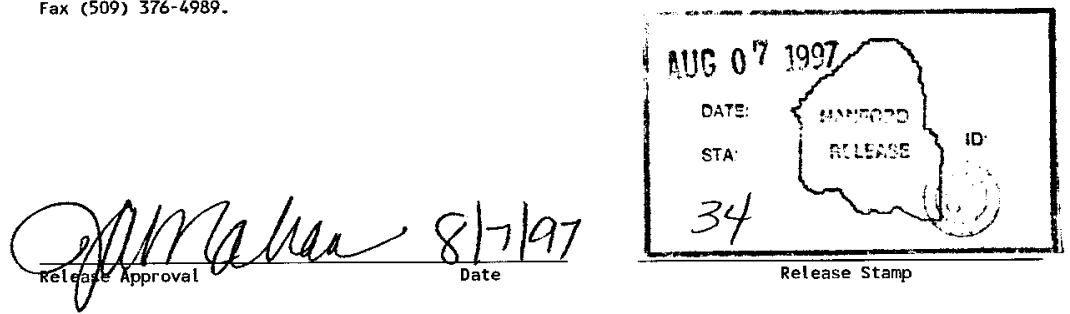

\section{Approved for Public Release}


Prepared For:

S.E.S. USA

1600 Kentucky St A3

Bellingham, WA 98226

\section{CALCULATIONS}

for the

40' Monopole

Hanford, Washington

October 17, 1996

Project No. 96828

Prepared By:

\section{LA HATT ENGINEERING CORPORATION}

P.O. BOX 5192

BELLINGHAM, WA 98227

(360) 676-8332

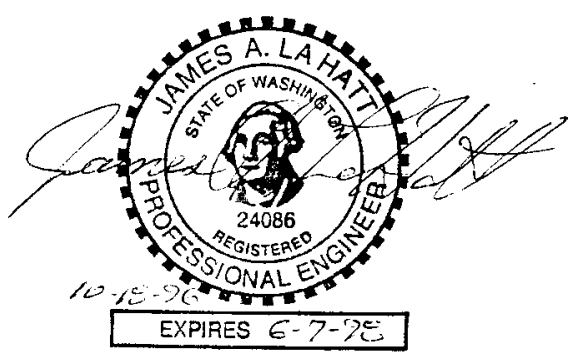


CLIENT LES LISA

PROJECT 40 MUNUPULE

BY SAL CK
LA HAT ENGINEERING

CORPORATION

P.O. Box 5192

Bellingham, WA 98227

676-8332

SCOPE OF WORK:

REVIEW CLIENTS 40 FT MONOPOLE DESIGN FOR STRUCTURAL ADEQUACY ASSUMING $90 \mathrm{mph}$, ExposurE "C" wimp LuADNC \& An ImportanCE FAC TR OH =1.15.

A 53 PIPE

A 36 SHApes \& PLATES

A.325 ButTS

A-307 Amender Butts

$f_{c}^{\prime}=2500$ pos

$f_{y}=60 k s i$

1700 psf Aclu' sol pressure

La Halt Engineering Corporation has been retained to provide services for only those items listed in the scope of work for this project and will not be held accountable for items not listed. For details of construction not shown, the provisions of THE PRSJELT SPECIFICATEAx shall form the minimum acceptable standards of construction. The design conclusions presented in this document are for use on this project only and are not intended for use on other projects without the written permission of La Hat Engineering Corporation. Our interpretations and conclusions are based on data made available to us by the Client. Should actual conditions vary from those addressed in our analysis, we should be provided the opportunity to reevaluate our findings in relation to the new data

Attach additional narrative sheets as required,

RECORD OF REVISIONS TO SCOPE:

Rev.

Date

By

Description

O $\quad 10-18-96 \quad \mathrm{JAL}$

ISSUES TS CLIENT 


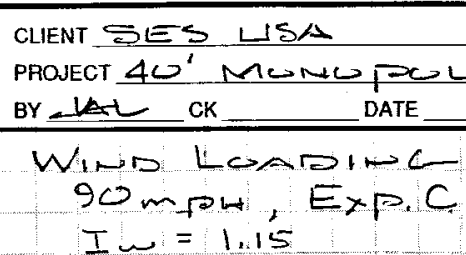

$$
\text { (n) }
$$

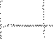
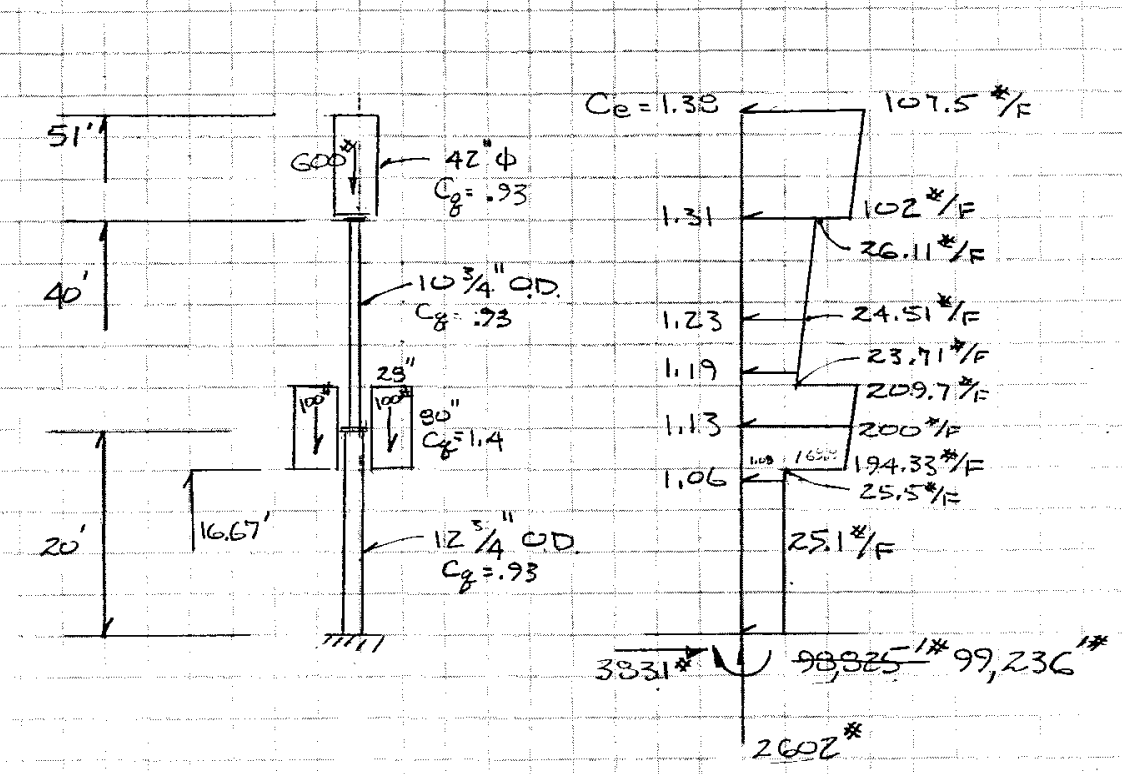


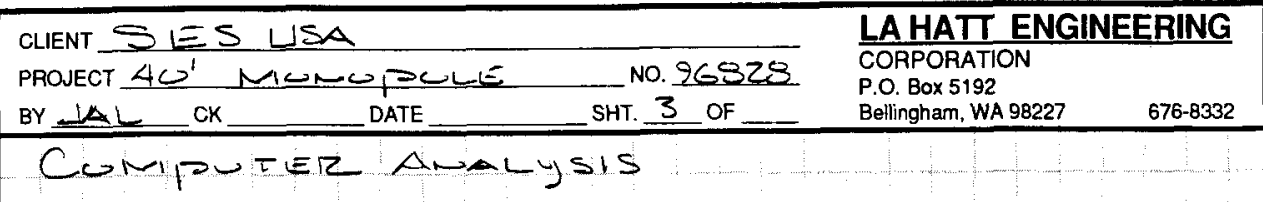

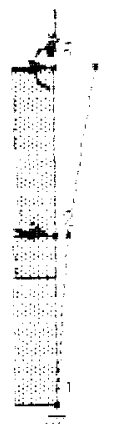

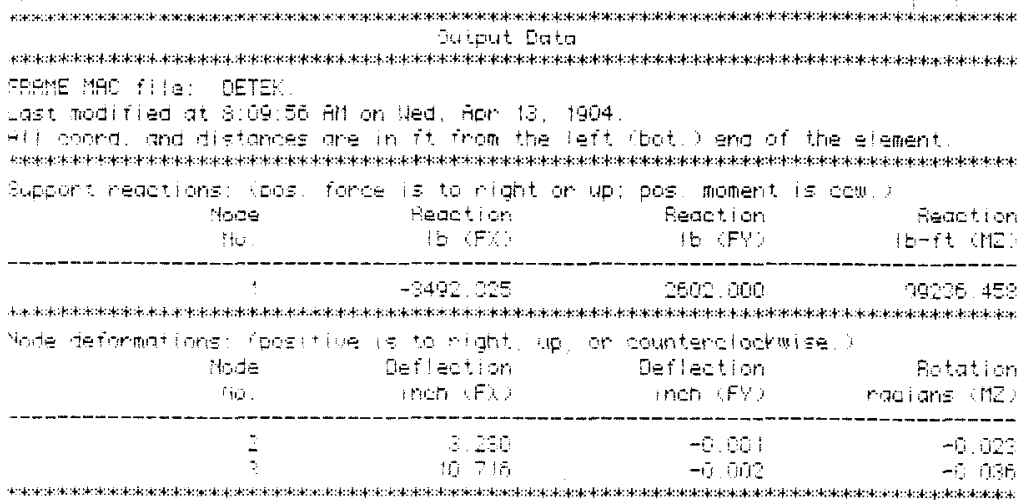

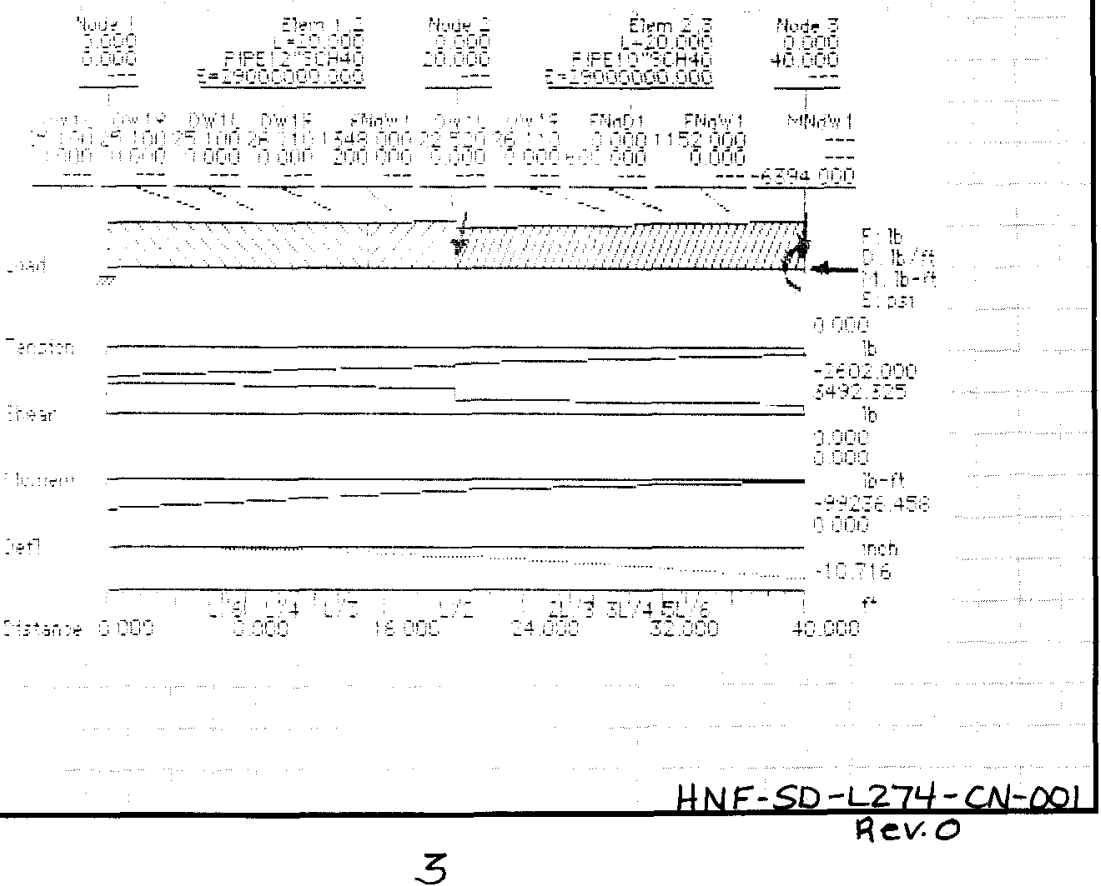




\begin{tabular}{|c|c|c|}
\hline LIENT SES & & LAHATT ENGINEERING \\
\hline PROJECT $40^{\circ}$ MSNSPC & No. 26828 & $\begin{array}{l}\text { CORPORATION } \\
\text { P.O. BOX } 5192\end{array}$ \\
\hline
\end{tabular}

Cumputerz Amamysis Continued

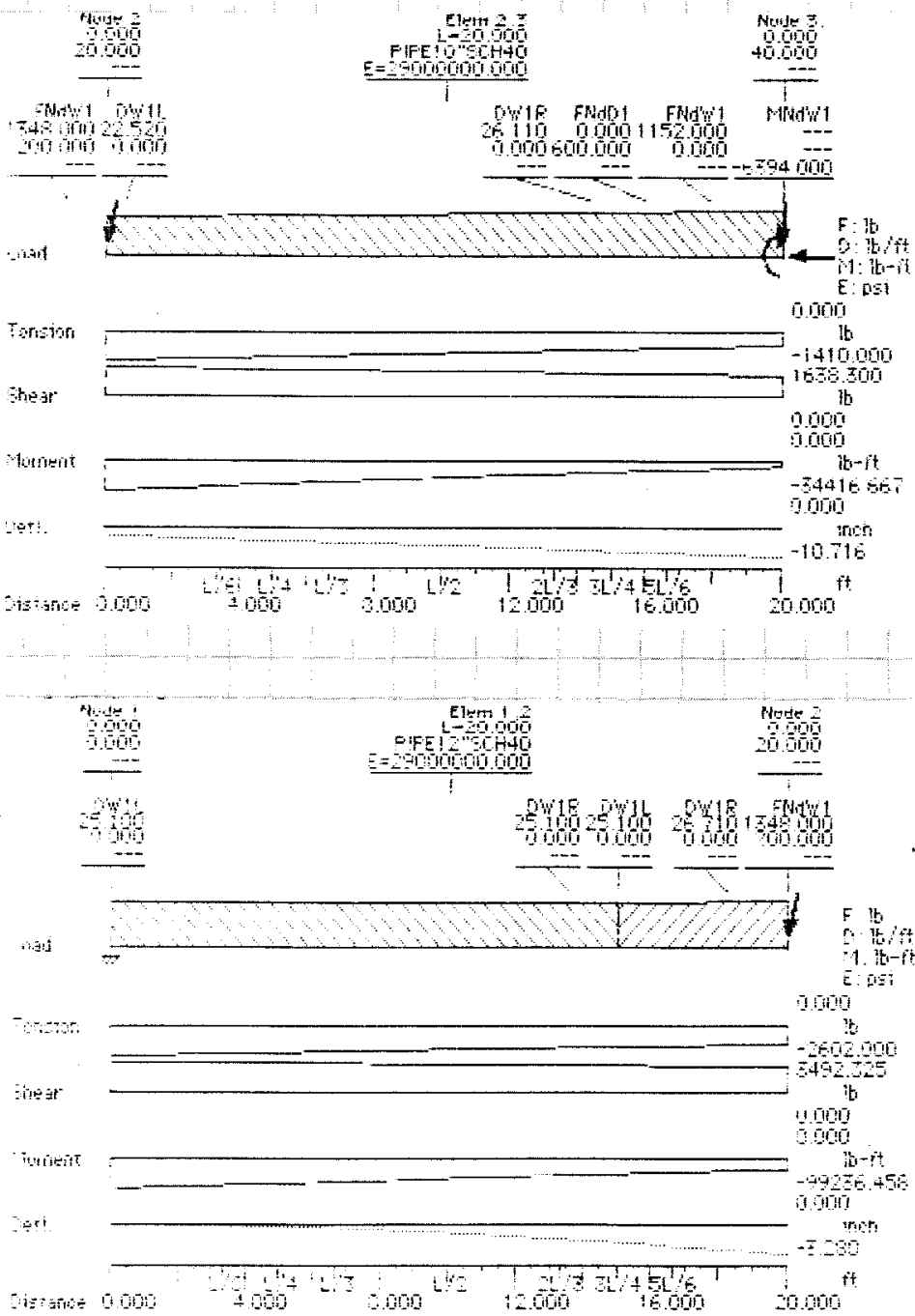


LA HATT ENGINEERING CORPORATION

P.O. Box 5192

Bellingham, WA 98227

$676-8332$

CHECK HPPER POLE

$10 " S C H 40$ pIPE; $S_{x}=29.91 \mathrm{~m}^{3}, A_{s}=11.91 \mathrm{in}^{2}, r=3.67$

$$
\begin{aligned}
& M_{2}=34,417^{\prime 4} \\
& M_{1}=6,34^{14} \\
& T^{\prime}=1410^{2} \\
& V=1638^{\$}
\end{aligned}
$$

$$
\begin{aligned}
& f_{a}=\frac{1410^{*}}{11.91}=119.4 \mathrm{ps} 1 \\
& C_{c}=\sqrt{\frac{2 \pi^{2} 29 \times 10^{6}}{35 \times 10^{3}}}=127.9<\frac{\mathrm{KL}}{r}=\frac{z(40 \times 12)}{3.67}=261.6 \\
& F_{a}=\frac{12 \pi^{2}\left(29 \times 10^{6}\right)}{23(261.6)^{2}}=2,132 \mathrm{psi} \\
& \frac{f_{a}}{F_{a}}=0.05<15 \% \quad \therefore U S=E Q \mathrm{HI}-3 \text { (arsc) }
\end{aligned}
$$

EY OBEERVATION $L_{b}>L_{C} \therefore F_{b}=.6 F_{y}=21 \mathrm{ks}$

$$
f_{b}=\frac{34,417^{1}(12)}{29.91 \mathrm{n}^{3}}=13,203 \mathrm{ps}
$$

$$
\frac{f_{a}}{F_{a}}+\frac{f_{b}}{F_{b}(1.33)}=\frac{110.4}{2102}+\frac{13,008}{21,000(1.33)}=0.55<10 k
$$

$$
\text { LSE } 10^{\prime \prime} \mathrm{SCH} 40
$$

HNF-SD-L274-CN-DOI 


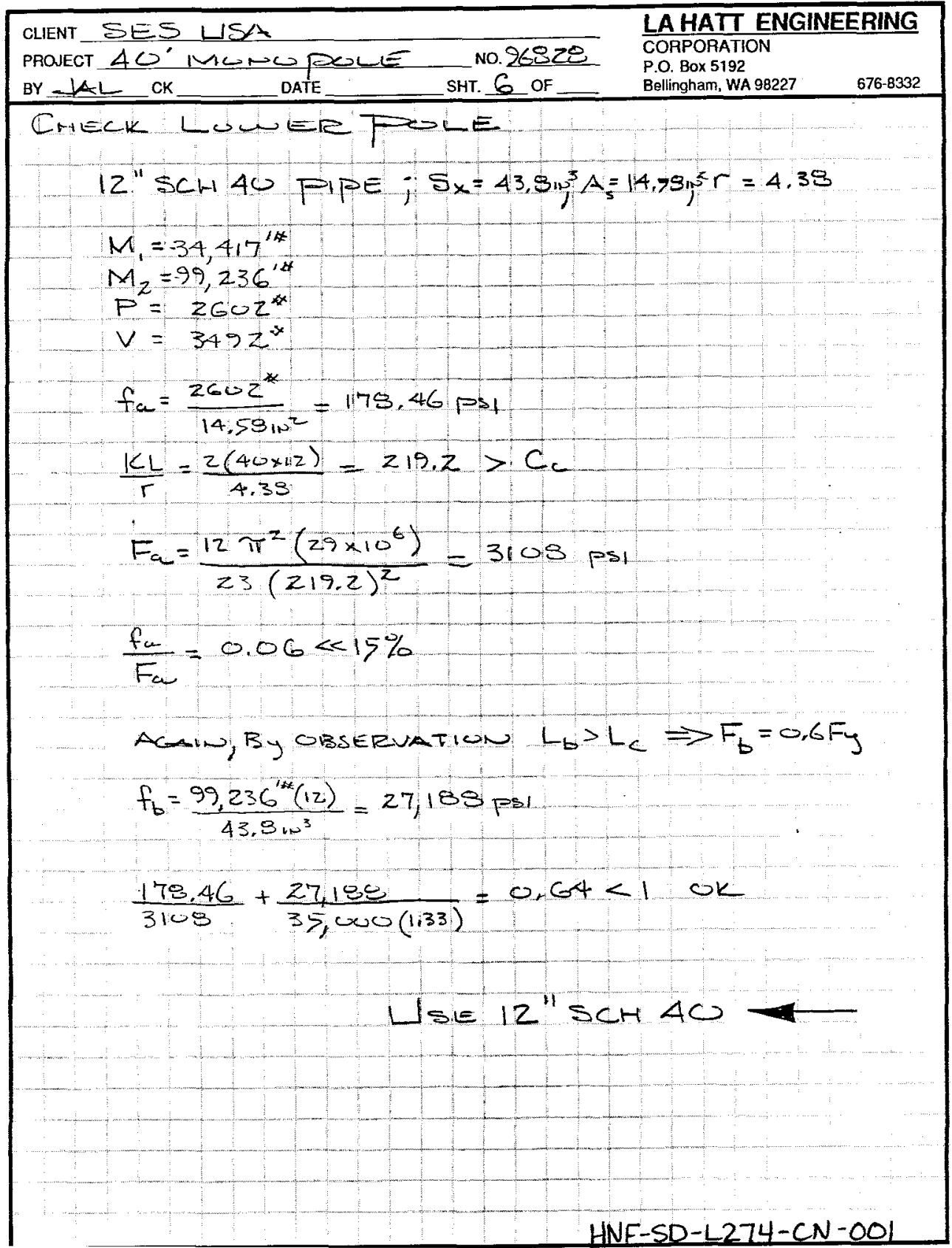




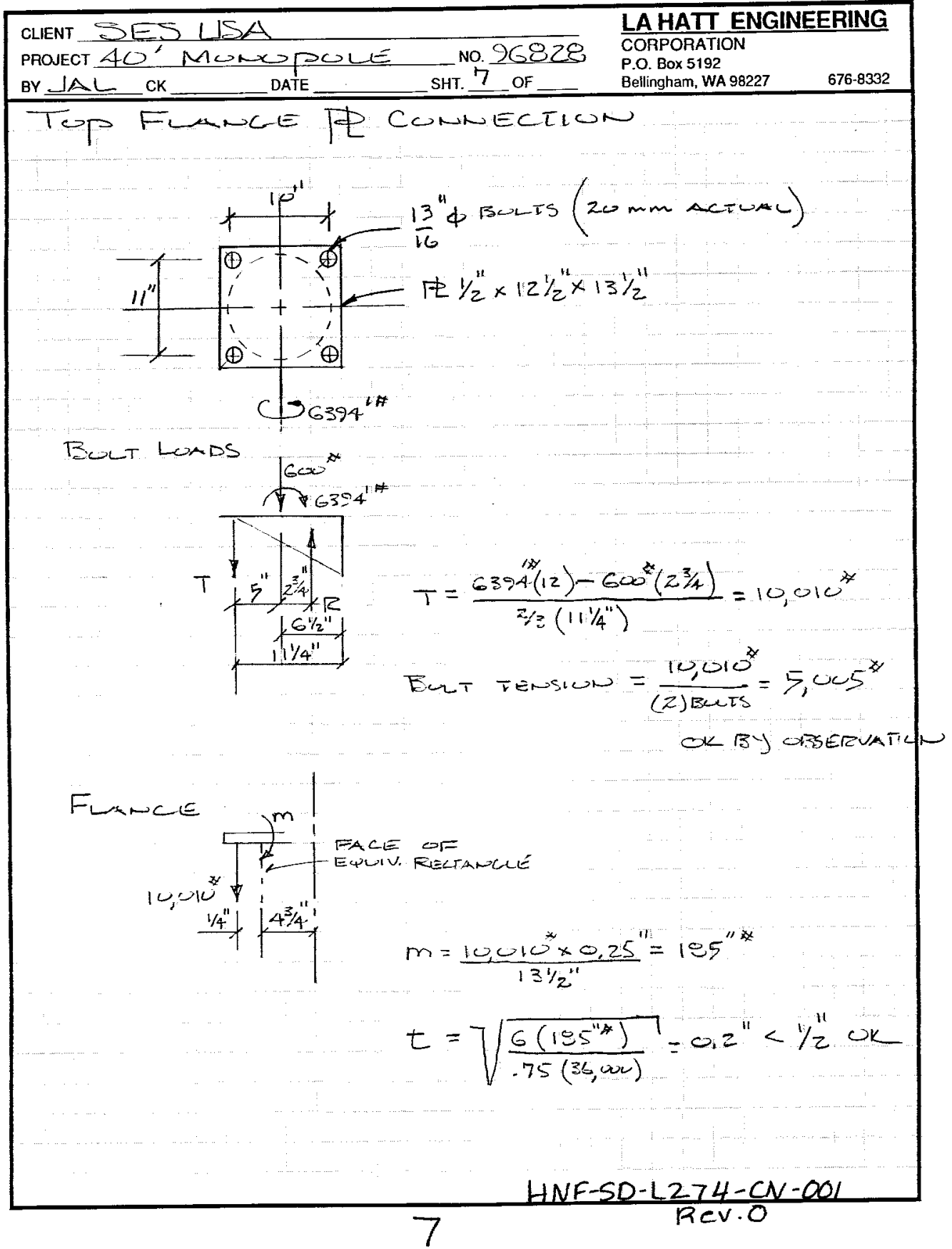




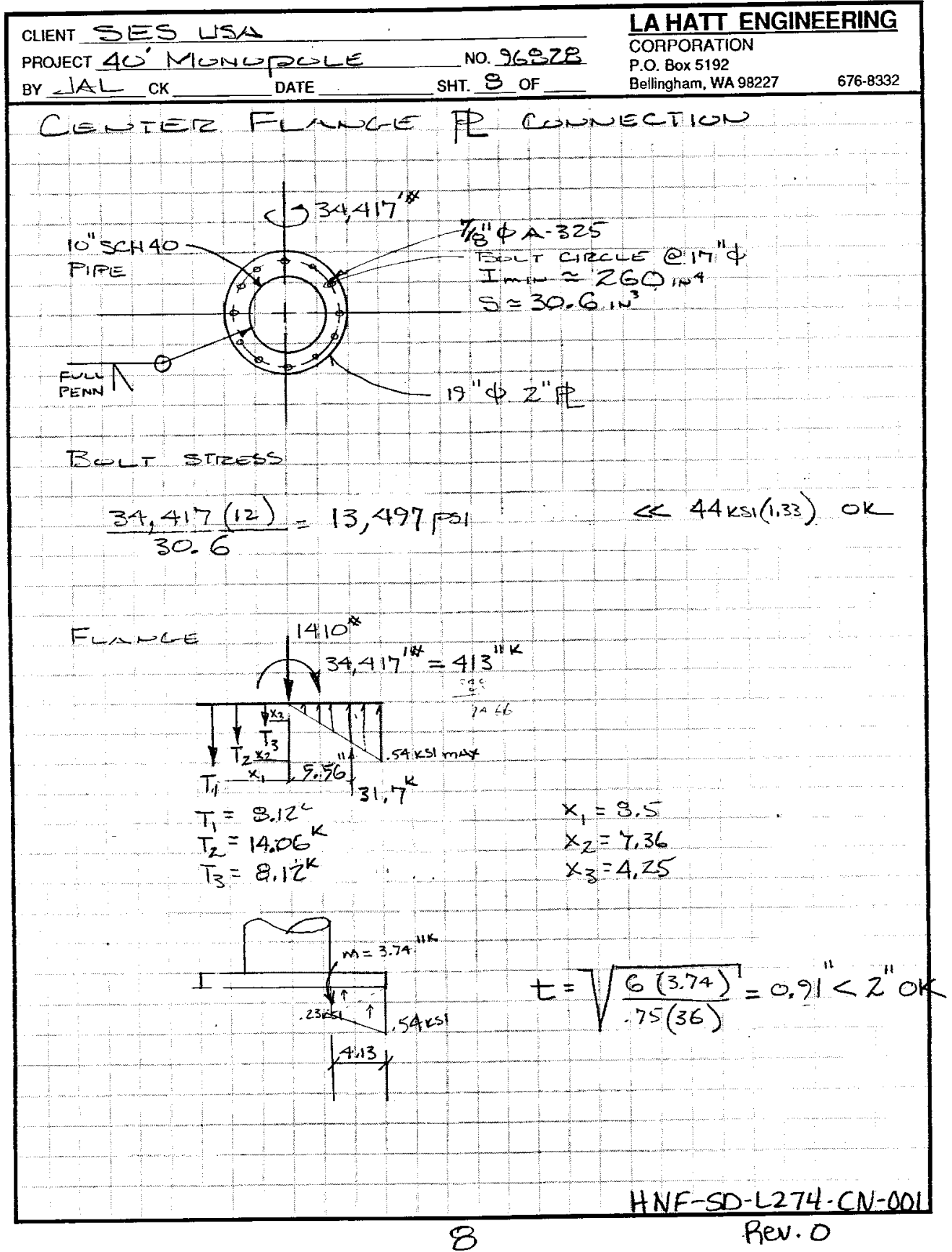




$$
\text { CLIENT SES USA }
$$

LA HATT ENGINEERING PROJECT $40^{\prime}$ MONIOPOLE NO. IGSZS CORPORATION $\begin{array}{lll}\text { P.O. Box } 5192 & \text { PF }\end{array}$

BOTtUM flance it CONNELTION

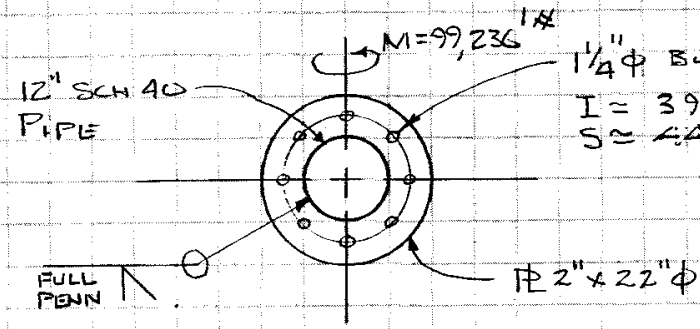

BULT STRESS

$$
\frac{99,236(12)}{44.3310^{3}}=26,963, p s 1<44 \mathrm{ks1}(1.33) \text { or }
$$

Functe

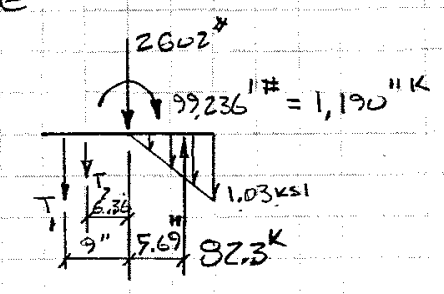

$$
\begin{aligned}
& T_{1}=33^{k} \\
& T_{2}=46.7^{k}
\end{aligned}
$$

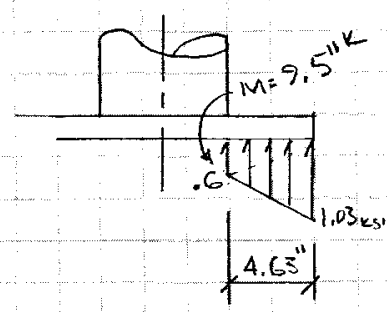

$$
t=\sqrt{\frac{6(9.5)}{75(36)}}=1.45^{11}<2^{\prime \prime} \mathrm{K}
$$

$$
\frac{H N F-S D-L 274-C N-D 01}{R E N . O}
$$

9 


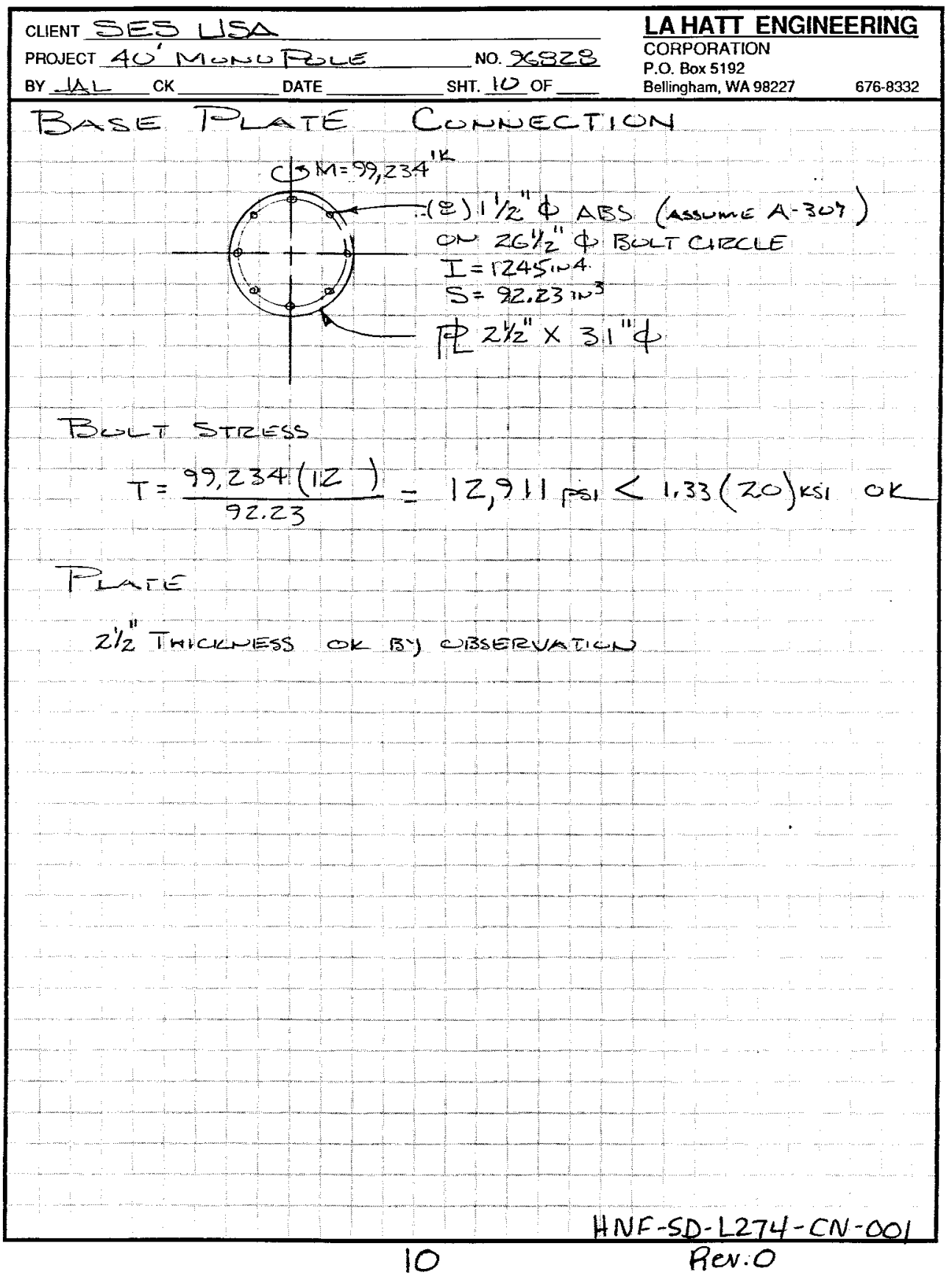


CLIENT BES LISA

PROJECT $40^{\prime}$ MONOPOLE NO. 96828

BY ISL CK DATE SHT. 11_ OF
LA HAT ENGINEERING CORPORATION

P.O. Box 5192

Bellingham, WA 98227

$676-8332$

TuUNAMTIUN

$$
\text { CHECK } 9^{\circ} \times 9^{\prime} \times 3.75^{\prime}
$$

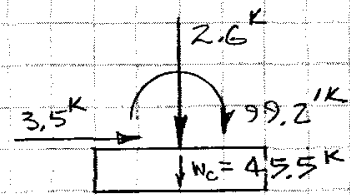

STABILITy

$$
\text { SLID }=\frac{.35\left[45.5^{k}+2.6^{k}\right]}{3.5^{k}}=4.8 \rightarrow 1.5 \text { ok }
$$

OVERTURN WC

$$
S F=\frac{[2.6+45.5] 4.5^{\prime}}{99.2^{1 k}+\left(3.5^{k} \times 3.75^{\prime}\right)}=1.93>1.50 k
$$

CONTACT PRESSURE

$$
\begin{aligned}
& e=\frac{99.2^{k}+\left(3.5^{k} \times 3.75^{\prime}\right)}{45.5^{k}+2.6^{k}}=2.34^{\prime}>\frac{B}{6} \\
& \therefore \sigma=\frac{2(N)}{3\left(\frac{B}{2}-e\right) L}=\frac{2\left(48.1^{k}\right)}{3\left(\frac{9}{2}-2.34\right) 9^{\prime}}=1.65 \mathrm{ksF} \\
& \therefore 1.33(1.5 \mathrm{kSF}) \text { ok }
\end{aligned}
$$

END. THICKNESS

Tuoway SHEAr

OK BY OBSERVATION

Flexural shear

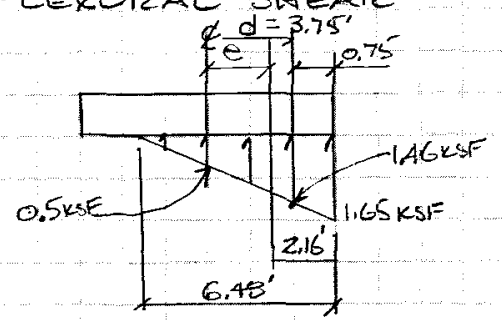

$$
\begin{aligned}
& V_{4}=1,7\left(\frac{1.46+1,65}{2}\right) 0.75^{\prime}\left(9^{\circ}\right)=17.94^{k} \\
& d_{\text {REQ D }}=\frac{17,940^{*}}{.35(2) \sqrt{2,500} 109^{41}}=1.9^{\prime \prime}
\end{aligned}
$$

ok

HNF-SD-L274-CN-DO1

II

Rev. 0 


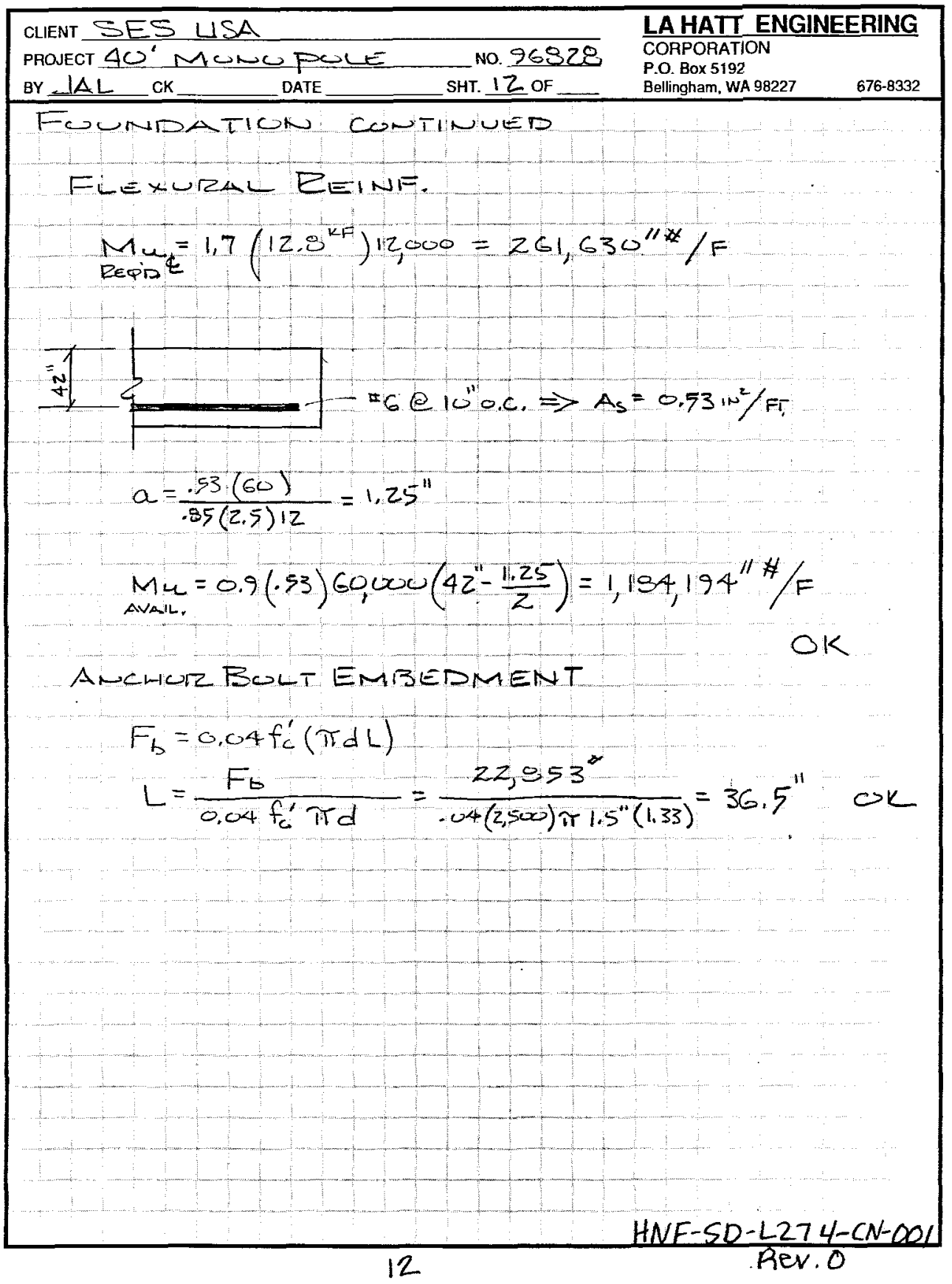




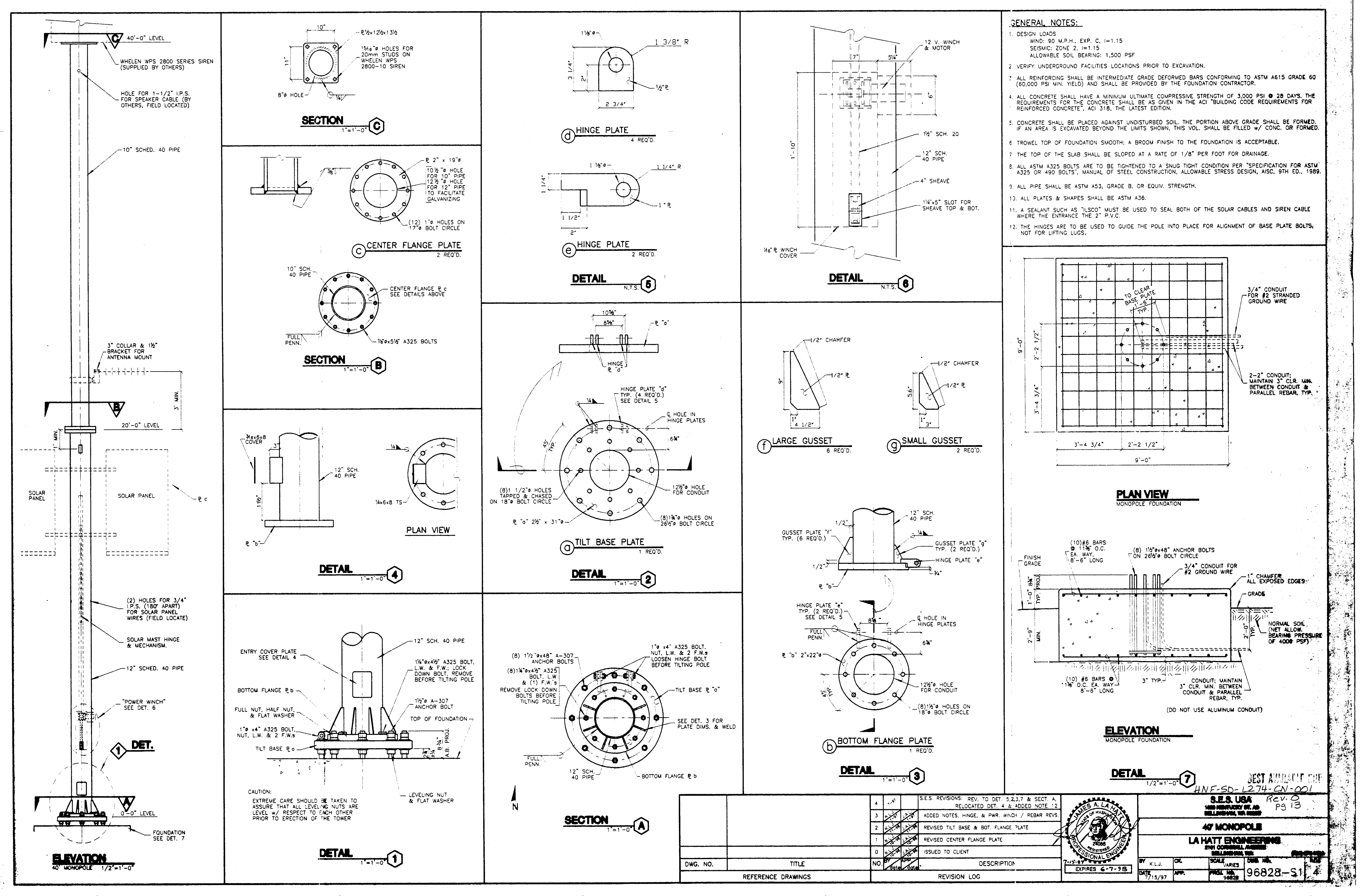

